\title{
Axial Flow Effect on the Stability of Circular Couette Flow
}

\author{
H. Belkadi ${ }^{1 \dagger}$, Y. Laghouati ${ }^{1}$, V. Sobolik ${ }^{2}$, H. Oualli ${ }^{3}$ and A. Bouabdallah ${ }^{4}$ \\ ${ }^{1}$ Laboratory of Modelisation and SImulation in Fluid Mechanics (LAMOSI), University of Sciences and \\ Technology of Oran, USTO-MB, 31000, Oran, Algeria \\ ${ }^{2}$ LaSIE, UMR 7356, University of La Rochelle, La Rochelle, France \\ ${ }^{3}$ Laboratory of Fluid Mechanics, Ecole Militaire Polytechnique (EMP), Borj El Bahri, 16111 Algiers, \\ Algeria \\ ${ }^{4}$ Laboratory of Thermodynamic and Energetical Systems (LTSE), USTHB, Bab Ezzouar, 16032 Algiers, \\ Algeria \\ †Corresponding Author Email: hichem.belkadi@univ-usto.dz
}

(Received June 17, 2021; accepted September 11, 2021)

\begin{abstract}
We investigate the effect of an axial Poiseuille annular flow on the stability of Taylor vortices via numerical simulation using CFD Ansys Fluent software. The working conditions are identical to those of the TaylorCouette experimental device of the LaSIE laboratory, where the inner cylinder is rotated. An incompressible fluid of density $\rho=998 \mathrm{~kg} / \mathrm{m}^{3}$, with a kinematic viscosity $v=1.004 * 10^{-6} \mathrm{~m}^{2} / \mathrm{s}$ at a temperature $\mathrm{T}=19.5^{\circ} \mathrm{C}$ is considered. The geometrical parameters of the flow system are characterized by a height $\mathrm{H}=275 \mathrm{~mm}$, a radius ratio $\eta=0.804$, and an axial aspect factor $\Gamma=45.45$. The axial Reynolds number and Taylor number are respectively in the ranges of $0 \leq \mathrm{R}_{e_{a x}} \leq 12$, and $0 \leq T a \leq 142.25$. Flow control is carried out according to two distinct protocols to bring out the effect of axial flow on the evolution of the Taylor vortex Flow (TVF). The first consists of superimposing an azimuthal flow around the critical TVF threshold with increasing axial flow until the Taylor vortices disappear. In the second, an axial field is set and the Taylor number is varied until onset of the TVF mode. It is predicted that in the presence of an axial flow, the critical threshold for first instability triggering (TVF) is delayed. In addition, the ratio of the axial phase velocity to the mean axial velocity of the axial base flow is 1.16. This value agrees well with previous results reported in literature.
\end{abstract}

Keywords: Taylor-Couette flow; Axial flow; Taylor vortex; Simulation.

\section{NOMENCLATURE}

$\begin{array}{ll}\mathrm{C}_{\mathrm{f}} & \text { skin friction coefficient } \\ \mathrm{d} & \text { annular gap } \\ \mathrm{H} & \text { height of cylinder } \\ \mathrm{k} & \text { axial wave number } \\ \mathrm{R}_{1} & \text { radius of Inner Cylinder (IC) } \\ \mathrm{R}_{2} & \text { radius of Outer Cylinder (OC) } \\ r, \theta, z & \text { cylindrical coordinates } \\ \mathrm{Re}_{\mathrm{ax}} & \text { axial Reynolds number } \\ \mathrm{Ta} & \text { Taylor number } \\ \mathrm{T}_{\mathrm{a}}^{*} & \text { Taylor number } \\ \mathrm{T}_{\mathrm{ac}}^{*}, \mathrm{~T}_{\mathrm{ac}_{1}} \text { critical Taylor number of the first } \\ \quad \quad \text { instability }\end{array}$

$\mathrm{T}_{\mathrm{ac} 2}$ critical Taylor number of the second instability $x$ dimensional radial distance

$V_{z} \quad$ axial velocity

$\eta \quad$ radius ratio

$\lambda$ axial wavelength

$v \quad$ kinematic viscosity

$\rho$ density

$\Gamma \quad$ aspect ratio

$\Omega_{1} \quad$ angular velocity of inner cylinder 


\section{INTRODUCTION}

The Taylor-Couette flow system represented by the motion of a viscous fluid between two coaxial cylinders, where only the inner cylinder rotates, is has been thoroughly studied in the past due to its the great impact on both fundamental and applied areas.

Theoretically, this simple system's configuration is chosen in order to better understand transition to turbulence. Several unsteady modes are identified such as: Taylor Vortex Flow (TVF), Wavy Vortex Flow (WVF), Modulated Wavy Vortex Flow (MWVF) and Taylor Turbulent Vortex Flow (TTVF). Practically, the Taylor-Couette system is encountered in various applied fields as in viscometry, filtration processes and industry.

Since the pioneering work of G. I. Taylor, huge amount of experimental and theoretical researches are devoted to the Taylor-Couette system. For a thorough scientific contributions in this area the readers are referred to Chandrasekhar (1961), and Drazin and Reid (1981). Other important experimental works contributed to elucidate the evolution of instability structures in the TaylorCouette system under the influencing factors in relation with geometry and rotating speed. Burkhalter et al. (1973) confirmed the theoretical work on the critical thresholds of different instability modes using a visualization technique. Fenstermacher et al. (1979) measured the spatiotemporal periodicities and velocity fields of the Taylor-Couette flow system using laser velocimetry (LDA). Bouabdallah (1980) highlighted the transition process to turbulence for different gaps using polarographic method and spectral analysis.

Andereck et al. (1986) presented a diagram where the observed regimes are reported versus Reynolds numbers of the inner and outer cylinders. S.T. Wereley et al. (1998) studied evolution of the critical thresholds by measuring the radial and azimuthal velocity components, using the PIV (Particle Image Velocimetry) technique.

The Taylor-Couette system in the presence of an axial hydrodynamic field (Taylor-CouettePoiseuille) is still attractive for many researchers due to applications met in various industrial processes such as: filtration processes, photocatalytic reactors, heat exchangers, rotating machines, plain bearings, etc.

DiPrima and Pridor (1979), Ng and Turner (1982), and Recktenwald et al. (1993) analyzed the effect of axial flow at low axial Reynolds numbers on the stability of the Taylor-Couette system for various gaps, in linear theory for the axisymmetric case. They confirmed that the onset of the TVF mode is delayed in the presence of an axial field. In addition, they showed that Taylor vortices propagate axially where the ratio of the axial phase velocity to the mean base axial velocity at the column inlet is about 1.17 .

The theoretical results of DiPrima and Pridor (1979), Ng and Turner (1982) and Recktenwald et al. (1993) were experimentally checked by Lueptow et al.
(1992) using visualization, and Tsameret and Steinberg (1994) with Laser Velocimetry (LDA) for low values of the axial Reynolds number.

Using PIV technique, Lueptow and Wereley (1999) measured radial and axial velocity fields in a TaylorCouette-Poiseuille system (TCP) for a radius ratio of $\eta=0.83$. A cartography of various regimes versus Taylor number and axial Reynolds number could be constructed. Hwang and Yang (2004) numerically verified the values measured Lueptow and Wereley (1999) for the same working conditions. Same regimes are identified, namely: Taylor vortex, wavy Taylor vortex, stationary spiral vortex, wavy spiral vortex and turbulent wavy vortex.

Monfared and Shirani (2016) studied experimentally and numerically the TCP flow system according to two different protocols. Direct protocol, where rotational speed is constant and the inverse protocol, where only the axial field is held fixed. It is found that for both protocols, the instability structures are different for the same values of Taylor and Reynolds numbers.

Recently, Kristiawan et al. (2019) measured experimentally the azimuthal and axial shear rates at the outer cylinder for various regimes of the TCP flow using the polarographic method.

Kataoka et al. (1977) obtained empirically by an electrochemical method, a relationship between the axial motion of Taylor vortices and the periodic heat and mass rates at the outer cylinder.

Ohmura et al. (2005) numerically and experimentally investigated classification of solid particles suspended in a TCP system. They showed that fine particles are trapped in vortices while the large ones are drained by the intra-vortex flow.

The present numerical work is devoted to study effect of an axial hydrodynamic field on a TaylorCouette flow for moderate Reynolds numbers $\mathrm{R}_{e_{a x}} \leq 12$. To be more specific, it is aimed at highlighting the influence of the axial flow on the main flow stability regarding the first instability mode: TVF.

To this goal, the study is elaborated into two steps. The first consists in imposing a fixed value of the Taylor number Ta and varying the axial Reynolds number Reax until total flow relaminarization, and the second is relevant to superimposing an axial Reynolds number while varying Taylor number until re-appearance of the first mode, TVF.

\section{NUMERICAL PROCEDURE}

\subsection{Working Conditions}

The geometrical and physical conditions of the system are selected regarding working conditions of the Taylor-Couette experimental device of the LaSIE laboratory (University of La Rochelle) where criteria for appearance of TVF and WVF instability modes are experimentally established. 
The constant physical properties at a temperature $\mathrm{T}=19.5{ }^{\circ} \mathrm{C}$, concerns an incompressible fluid of density $\rho=998 \mathrm{~kg} / \mathrm{m}^{3}$, with a kinematic viscosity $v=$ $1.004 * 10^{-6} \mathrm{~m}^{2} / \mathrm{s}$. The working conditions are such that, the inner cylinder of radius $R_{1}=24.85 \mathrm{~mm}$ rotates with an angular velocity $\Omega 1$, and the outer cylinder of radius $R_{2}=30.9 \mathrm{~mm}$ is held fixed. The height of the flow system is $\mathrm{H}=275 \mathrm{~mm}$, the radius ratio $\eta=0.804$ and the aspect ratio is $\Gamma=45.45$.

In the present work, the Taylor number is defined as $\mathrm{Ta}=\frac{\Omega_{1} R_{1} d}{v}$ (Lueptow and Wereley 1999) although in the literature, we can find another expression for the definition of Taylor number as $T_{a}^{*}=\frac{\Omega R_{1} d}{v} \sqrt{\frac{d}{R_{1}}}$.

\subsection{Geometrical Configuration}

Numerical simulations are implemented on ANSYS Fluent software, with a structured hexagonal mesh (Fig. (1)) in the radial, azimuthal, and axial directions $(\mathrm{r}, \theta, \mathrm{z})$. Several mesh configurations are tested. Comparison of the obtained numerical values for critical characterizing values (transition to TVF and WVF) in terms of Taylor and Reynolds numbers with those in literature allowed to validate the adopted mesh with 772,800 cells $(13 * 200 * 275)$.

Pressure is discretized based on a second-order scheme. A third-order MUSCL scheme is used to discretization of the momentum equations. The hydrodynamic field Pressure-Velocity is coupled using PISO scheme. The convergence criterion is fixed to $10^{-5}$, and the time to $\Delta t=0.0002 \mathrm{~s}$.

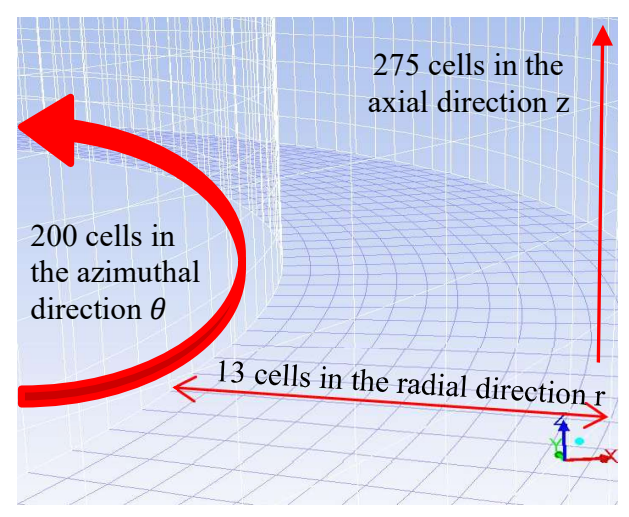

Fig. 1. Mesh configuration.

\subsection{Governing Equations}

The hydrodynamic field is represented by the velocity field $\vec{V}\left(V_{r}=v_{r}, V_{\theta}=\overline{V_{\theta}}+v_{\theta}, V_{z}=\bar{V}_{z}+v_{z}\right)$, and pressure $=\bar{P}+p$. Equations of motion in cylindrical coordinates $(r, \theta, z)$ are expressed as,

\section{Continuity equation}

$\nabla \cdot \vec{V}=0$

\section{Momentum equation}

$\frac{\partial \overrightarrow{\mathrm{V}}}{\partial \mathrm{t}}+(\overrightarrow{\mathrm{V}} \cdot \nabla) \overrightarrow{\mathrm{V}}=-\frac{1}{\rho} \nabla \mathrm{p}+v \Delta \overrightarrow{\mathrm{V}}$

Where,
$\Delta=\nabla^{2}=\frac{\partial^{2}}{\partial r^{2}}+\frac{1}{r} \frac{\partial}{\partial r}+\frac{1}{r^{2}} \frac{\partial^{2}}{\partial \theta^{2}}+\frac{\partial^{2}}{\partial z^{2}}$

is the Laplacien operator in cylindrical coordinates.

Boundary conditions associated to mean velocity field :

$\bar{V}_{\theta}=\bar{V}_{z}=0$, for $r=R_{2}$, and $\vec{V}_{\theta}=R_{1} \Omega_{1}$, for $r=R_{1}$.

Boundary conditions associated to perturbed velocity field :

$\mathrm{v}_{\theta}=\mathrm{v}_{\mathrm{r}}=\mathrm{v}_{\mathrm{z}}=0$, for $\mathrm{r}=\mathrm{R}_{1}$ and $\mathrm{r}=\mathrm{R}_{2}$.

\section{RESULTS AND DISCUSSIONS}

Evolution of the first TVF transition structure in presence and absence of an axial flow is considered. Specifically, results for the T-C system without an axial field (standard case) are first presented, then effect of the axial hydrodynamic field is considered.

\subsection{Flow without Axial Hydrodynamic Field $\left(\operatorname{Re}_{a x}=0\right)$}

\subsubsection{Appearance of TVF and WVF mode}

\section{a)- First instability mode TVF}

For transition to TVF mode, the critical numerical value is found equal to $\mathrm{T}_{\mathrm{ac}_{1}}^{*}=45.8$. This compares well to experiments of Recktenwald et al. (1993), Ali et al. (2002) where the presented value is $\mathrm{T}_{\mathrm{ac}_{1}}=98.43$ corresponding to a deviation of $5.6 \%$.

Figure 2 shows the first instability structure TVF in the vicinity of the critical threshold. The axial wavenumber $\mathrm{k}=23$ corresponds to a wavelength $\lambda=$ $2 \mathrm{~d}=12.5 \mathrm{~mm}$. This value is consistent with those from the literature. The value of the critical Taylor number for the TVF mode obtained numerically agrees well with critical value of Taylor number reported in literature as shown by table1.

\begin{tabular}{|c|c|c|}
\hline Authors & $\begin{array}{c}\text { Critical Taylor } \\
\text { number } \mathrm{T}_{\mathrm{ac}_{1}}\end{array}$ & $\begin{array}{c}\text { Precision } \\
\mathbf{( \% )}\end{array}$ \\
\hline $\begin{array}{c}\text { Recktenwald } \text { et } \\
\text { al. (1993) }\end{array}$ & 94.7331 & 1.9973 \\
\hline Ali et al. (2002) & 94.7336 & 1.9978 \\
\hline Present work & 92.8409 & \\
\hline
\end{tabular}

Table 1. Critical Taylor number evaluated by authors for $\eta=0.8$.

\section{b) - Second mode of instability WVF}

Figure 3 shows the structure of WVF instability in vicinity of the critical threshold. It is noted that the critical Taylor number $\mathrm{T}_{\mathrm{ac}_{2}}^{*}=73.88$, coincides well with the experimental critical value $\mathrm{T}_{\mathrm{ac}_{2}}^{*}=72.40$. This mode is characterized by the azimuthal undulation of the Taylor vortices. 


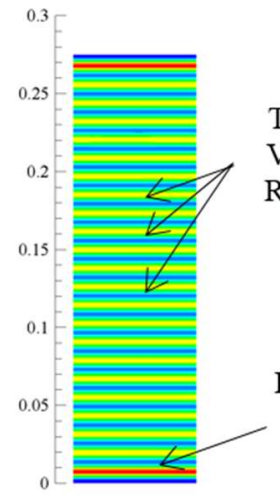

(a) $\mathrm{T}_{\mathrm{ac}_{1}}^{*}=45.8$

$\left(\mathrm{T}_{\mathrm{ac}_{1}}=92.84\right)$

Fig. 2. Representation of TVF mode in the vicinity of critical threshold: (a) Numerical study, (b) Experimental visualization.
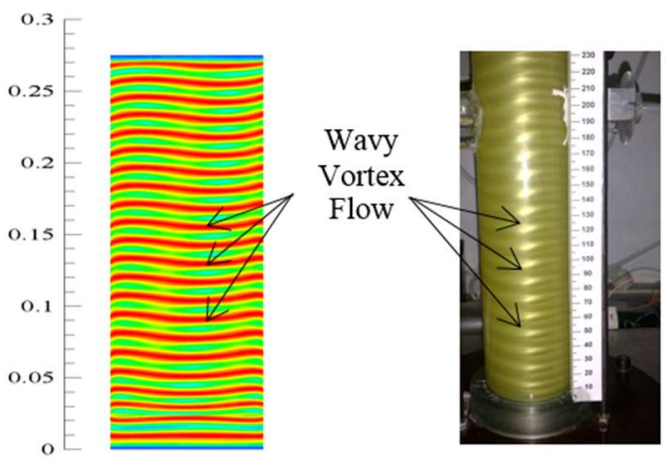

(a) $\mathrm{T}_{\mathrm{ac}_{2}}^{*}=73.88$

(b) $\mathrm{T}_{\mathrm{ac}_{2}}^{*}=72.40$

Fig. 3. Visualization of WVF mode in the vicinity of critical threshold: (a) Numerical study, (b) Experimental visualization.

In the experiment conducted by Coles(1965), considering a radius ratio $\eta=0.874$ and an aspect ratio $\Gamma=27.9$, the critical Taylor number of the second transition $\mathrm{T}_{\mathrm{ac}_{2}}$ is estimated to be about $55 \%$ above $\mathrm{Tc}_{1}$. which is in line with our results.

In our case, the critical threshold is evaluated at a $61 \%$ above $\mathrm{T}_{\mathrm{ac}_{1}}^{*}$. Appearance of the WVF wave observed experimentally corresponds to a rate of about $49 \%$ above $\mathrm{T}_{\mathrm{ac}}^{*}$.

\subsubsection{Transition from the stable laminar regime to the first instability structure}

Figure 4 shows details on the transition mechanism from laminar regime to the first TVF mode. An Ekman vortex signature is observed at the bottom and top borders for a low Taylor number value $\mathrm{T}_{\mathrm{a}}^{*}=$ 0.73 . When increasing the control number until the value $\mathrm{T}_{\mathrm{a}}^{*}=7.38$, a pattern of vertical isobaric lines is observed along the entire height. From $\mathrm{T}_{\mathrm{a}}^{*}=22.16$, a vertical wave train azimuthally launches in the system. At $\mathrm{T}_{\mathrm{a}}^{*}=36.94$, the formation of the Ekman vortex and the appearance of the first Taylor vortices at the top and bottom borders of the system. When increasing the Taylor number up to $\mathrm{T}_{\mathrm{a}}^{*}=44.33$, propagation of Taylor vortices progresses symmetrically from system endcaps towards the center. Finally, piled vortices occupy the entire height of the column at $\mathrm{T}_{\mathrm{a}_{\mathrm{c}}}^{*}=45.8$, characterizing the first mode of TVF instability.

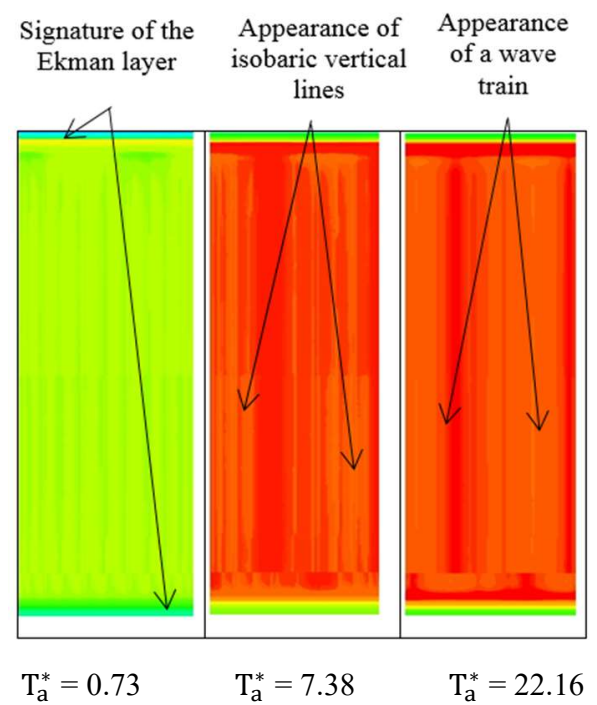

Ekman vortex developed Progressive piling of + appearance of the first Taylor vortices towards Taylor vortex the center
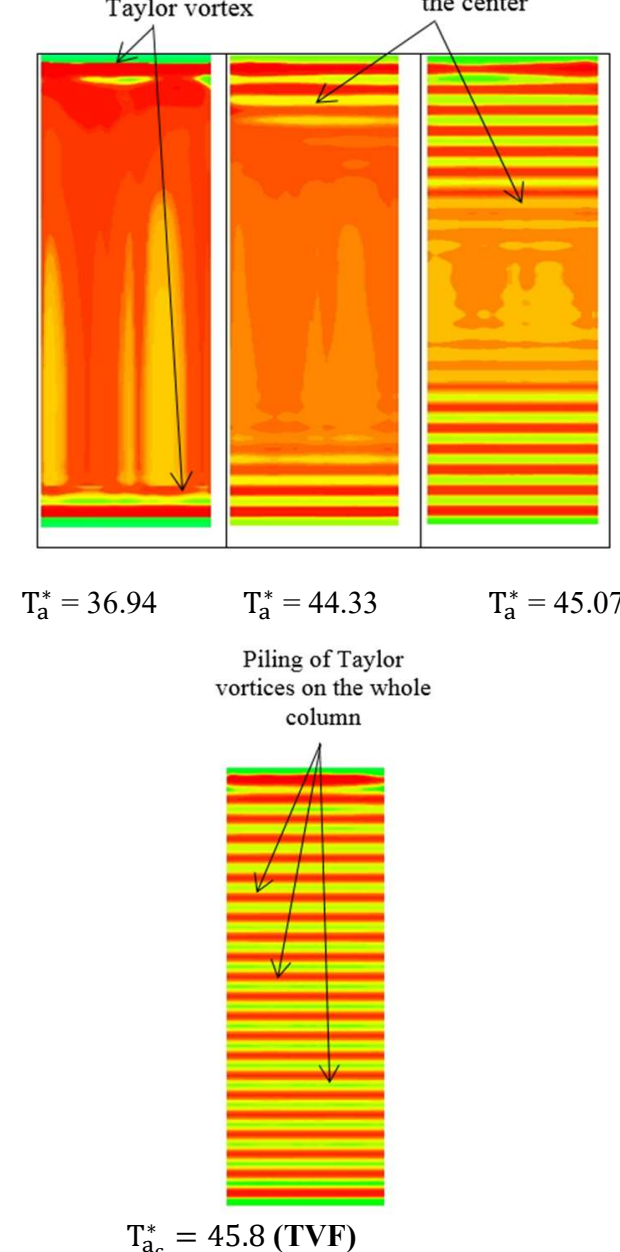

Fig. 4. Development of the centrifugal vortices from laminar regime to TVF mode. 


\subsubsection{Velocity and vorticity field distribution for TVF mode}

\section{a) - Velocity field}

Figure 5 depicts distribution of the radial, azimuthal and axial velocity fields. The radial velocity field (Fig. 5(a)) shows between the two counter-rotating cells, an alternation of positive maximum values (outflow) and negative (inflow). The maximum radial velocity is most important at the outflow as show in legend by maximum velocity contours $\mathrm{V}_{\mathrm{r}}=$ $7.89 \mathrm{E}-5 \mathrm{~m} / \mathrm{s}$. The outflow and inflow regions are shown in Fig. 5(d). Figure 5(b) exhibits repartition of the tangential velocity, where the maximum velocity is located near the inner wall and the minimum velocity is located next to the outer wall. The noticed undulations are due to the effects of inflow and outflow zones. Figure 5(c) indicates a shifted alternation, with respect to the central axis of the gap, of the maximum and minimum values of the axial velocity field. For a radial line passing through the center of the vortex, the axial velocity reaches a maximum value at a distance $r_{1}=0.25 \mathrm{~d}$ and a minimum velocity at $\mathrm{r}_{2}=0.75 \mathrm{~d}$ by passing through a zero velocity at $r_{3}=0.5 d$ (Fig. 14a).

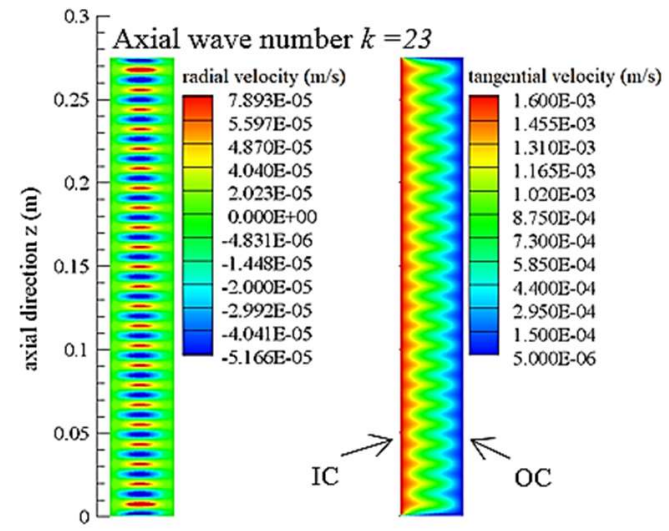

(a)

(b)

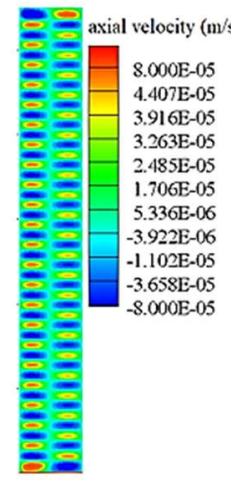

(c)

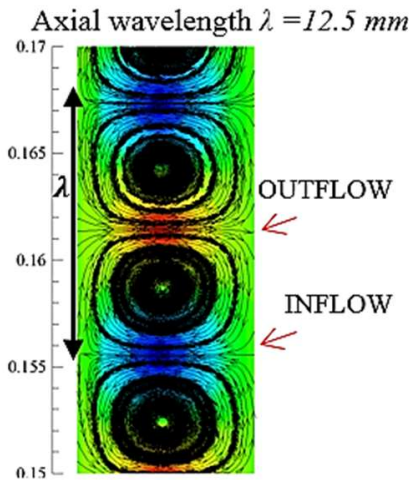

(d)
Fig. 5. Radial, azimuthal and axial velocity field components of the TVF mode.

\section{b) - Vorticity field}

Distribution of the vorticity field in the azimuthal plane is shown in Fig. 6. It can be noticed on Fig. 6(a), that the maximum positive and negative radial vorticity field values are superposed near the center of the gap. In the vicinity of the inner and outer walls, the radial vorticity is less important. Figure 6(b) shows the superposition of the maximum positive and negative values of the tangential vorticity at the central region of the gap and in a thin region near the inner wall side.

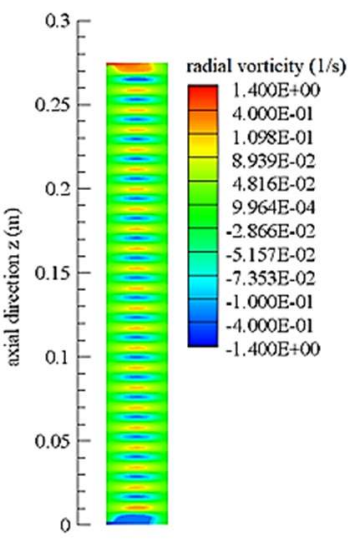

(a)

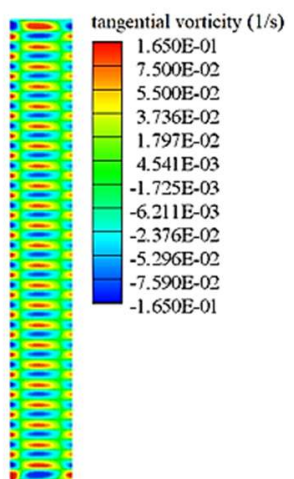

(b)

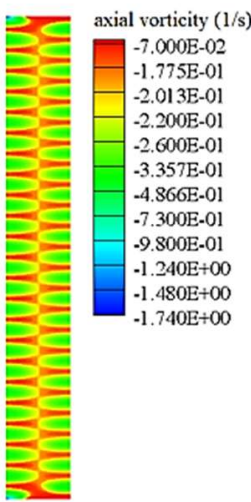

(c)

Fig. 6. Distribution of the radial, azimuthal and axial components of the vorticity field for the TVF mode.

We notice on Fig. 6(c), the superposition of the mean and minimum negative values on both sides of the central axis of the gap.

\subsection{Taylor-Couette Flow with Axial Flow $\left(\mathbf{R e}_{\mathrm{ax}} \neq \mathbf{0}\right)$}

To highlight the effect of the axial hydrodynamic field on the stability of the Taylor-Couette flow, this part of the study is carried out into two steps to concerning the first protocol In the first, a critical threshold Taylor number ( $\mathrm{T}_{\mathrm{a}}^{*}=45.8$ ) is set, and the axial Reynolds number is varied until the regime becomes completely stable. The second step consists in imposing a value for the axial Reynolds number, while progressively increasing the Taylor number value until the first instability mode onset. Two fixed values of the axial Reynolds number were chosen, namely, $\mathrm{Re}_{\mathrm{ax}}=6$ and $\mathrm{Re}_{\mathrm{ax}}=12$ in order to compare the values obtained by those chosen by Kristiawan et al. (2019). 


\subsubsection{Effect of the axial flow field on the Taylor- Couette flow}

The first protocol is applied in order to better follow evolution of the flow until vortices disappearance. Abdelali et al. (2019) used a technique to make the Taylor vortices disappear by combining the effect of the radial deformation of the inner cylinder with the oscillation of the free surface. The vortices disappear from the free surface in the depth direction. Abdelali et al. (2019) by numerical and experimental study, concluded that the Taylor vortices can easily be destroyed using low deforming frequencies, but The Ekman cells requires a high frequency to be destroyed.

The axial flow technique seems to lead to similar results but in the opposite direction. The strength of this technique lies in the fact that it destructs the vortices without resorting to the free surface and thus the system can be completely filled. This is not possible with radial deformation.

\section{a) - Distribution of Taylor vortex versus the axial Reynolds number}

Figure 7 shows the influence of the axial field on the stability of the flow and in particular the partial and total relaminarization versus the control number $\mathrm{Re}_{\mathrm{ax}}$. We notice that for the value $\mathrm{Re}_{\mathrm{ax}}=0.6$, the Taylor vortices occupy the whole column and no alteration is detected in the flow configuration. By fixing the value $\operatorname{Re}_{\mathrm{ax}}=3$, we observe a partial disappearance of the piling in the region $0<\mathrm{z} \leq 45$ $\mathrm{mm}$. From $\mathrm{Re}_{\mathrm{ax}}=6$, the piling vortices becomes limited to a region above the upper half of the column $(180<\mathrm{z} \leq 275 \mathrm{~mm})$. The flow becomes completely stable for a value of $\mathrm{Re}_{\mathrm{ax}}=12$, Taylor vortices completely disappear and excepted Ekman structures which subsist at the system endcaps. The flow is quasily relaminarized elswhere.

\section{b) - Stream lines and azimuthal vorticity distribution}

Figure 8 represents the azimuthal vorticity distribution for different values of the axial Reynolds number. It is seen in Fig. 8(a) that an axial ascendant flow evolves between the contra-rotating Taylor vortices from bottom to top of the system. The upward flow at the base of the flow system tends to increase the size of the first two contra-rotating cells, and reach relatively to the weak value $\mathrm{Re}_{\mathrm{ax}}=0.6$ an axial wavelength $\lambda=20 \mathrm{~mm}$. It is observed in Fig. $8(\mathrm{~b})$, that for the value $\mathrm{Re}_{\mathrm{ax}}=3$, the Taylor vortices disappear in the region $0<\mathrm{z} \leq 45 \mathrm{~mm}$ as revealed in Fig. 7. The vortices are separated in such a way that, the positive vortices are oriented towards the inner wall, while the negative vortices are deflected towards the outer wall. The axial wave number over the entire column reduces to $\mathrm{k}=19$. We note in Fig. 8(c) that for $\mathrm{Re}_{\mathrm{ax}}=6$, the relaminarization (straight streamlines) of the flow exceeds half the axial height $(\mathrm{z}=180 \mathrm{~mm})$ and the wave number reduces to $\mathrm{k}=8$. In the region $180 \mathrm{~mm}<\mathrm{z} \leq 275 \mathrm{~mm}$, the streamlines are undulated. As shown in Fig. 8(d), flow is completely relaminarized for $\mathrm{Re}_{\mathrm{ax}}=12$. The contrarotating cells vanish completely, which is consistent with the experimental results performed by Lueptow et al. (1992).
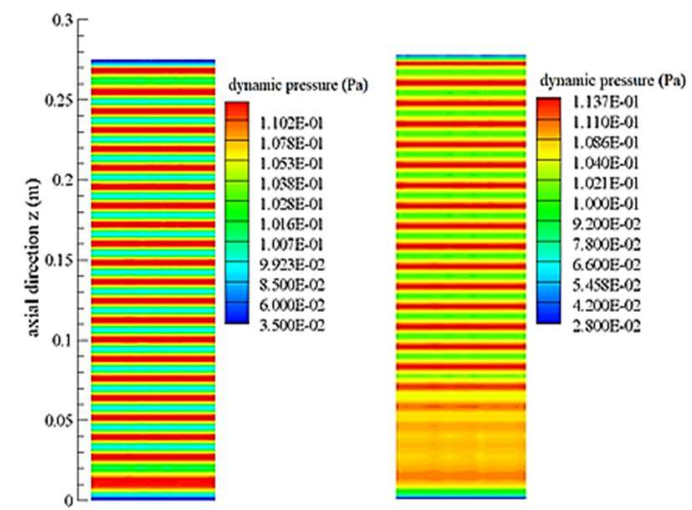

$\mathrm{Re}_{\mathrm{ax}}=0.6, \mathrm{Ta}=92.84$

$\operatorname{Re}_{\mathrm{ax}}=3, \mathrm{Ta}=92.84$

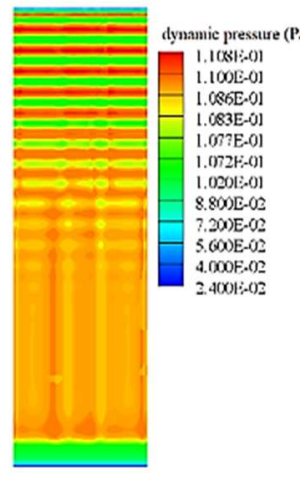

$\mathrm{Re}_{\mathrm{ax}}=6, \mathrm{Ta}=92.84$

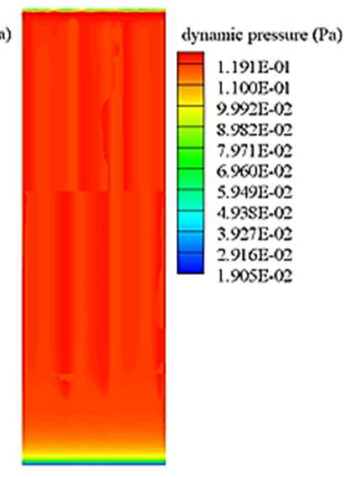

$\mathrm{Re}_{\mathrm{ax}}=12, \mathrm{Ta}=92.84$
Fig. 7. Evolution of TVF mode for different values of the axial Reynolds number.

Figure 9 shows the axial distribution of the friction coefficient at the outer cylinder versus the axial Reynolds number $R_{\text {ax. }}$ It appears that in the presence of the axial field the intensity of the Ekman peak attenuates at the bottom border.

As shown in Fig. 9(a), the friction coefficient varies periodically between the minimum value $\mathrm{C}_{\mathrm{f}} \min =$ 0.0027 and the maximum value of the skin coefficient $C_{f \max }=0.0063$.It is observed in Fig. 9(b) that for an axial Reynolds number of $\mathrm{Re}_{\mathrm{ax}}=3$, the optimal peak intensities are reduced respectively to $\mathrm{C}_{\mathrm{f} \min }=0.0028$ and $\mathrm{C}_{\mathrm{f} \max }=0.0058$. Accessing the value $\mathrm{Re}_{\mathrm{ax}}=6$, we notice in Fig. 9(c) that the peaks decay around the values $\mathrm{C}_{\mathrm{f} \text { min }}=0.0037$ and $\mathrm{C}_{\mathrm{f} \max }=$ 0.0047 in the axial region along $0.18<z \leq$ $0.275 \mathrm{~m}$.

\section{c) - Distribution of friction coefficient in function of the axial Reynolds number}

In the bottom axial region: $z \leq 0.18 \mathrm{~m}$, a fixed value of the friction coefficient $\mathrm{C}_{\mathrm{f}}=0.0042$ is recorded. As shown in Fig. 9(d), relatively to the value $\mathrm{Re}_{\mathrm{ax}}=12$, the friction coefficient becomes constant along the outer cylinder $\mathrm{C}_{\mathrm{f}}=0.005$. 


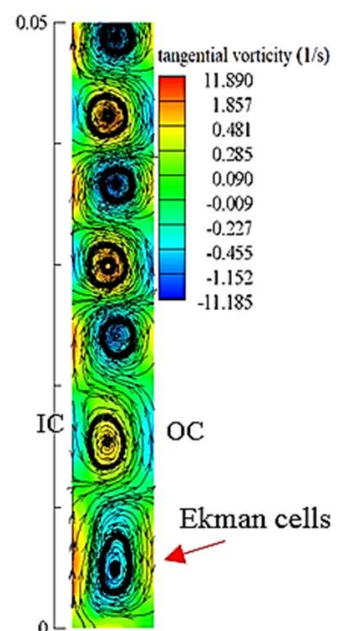

(a)

$\mathrm{Re}_{\mathrm{ax}}=0.6, \mathrm{Ta}=92.84$

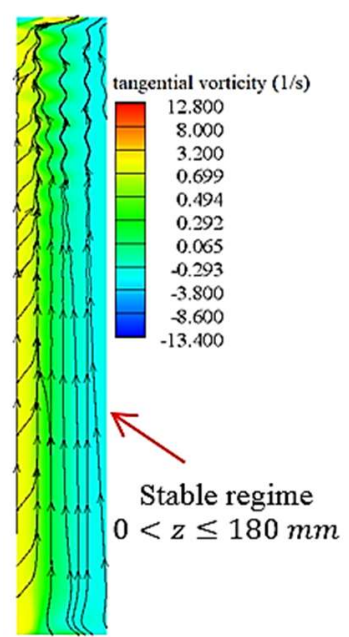

(c)

$\mathrm{Re}_{\mathrm{ax}}=6, \mathrm{Ta}=92.84$

(d)

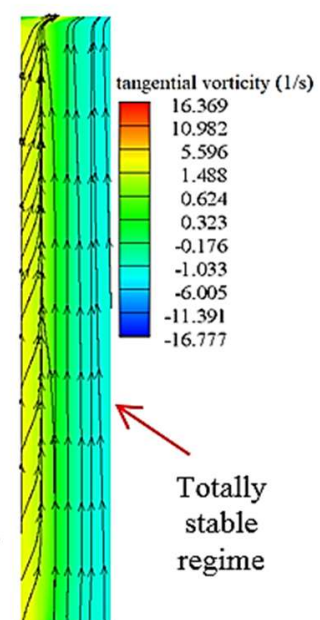

$\mathrm{Re}_{\mathrm{ax}}=12, \mathrm{Ta}=92.84$

Fig. 8. Streamlines and azimuthal vorticity distribution versus axial Reynolds number.

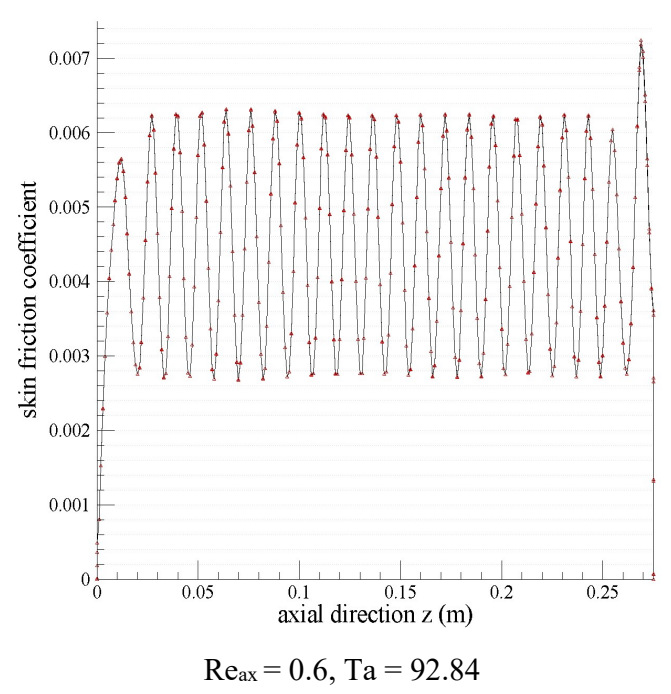

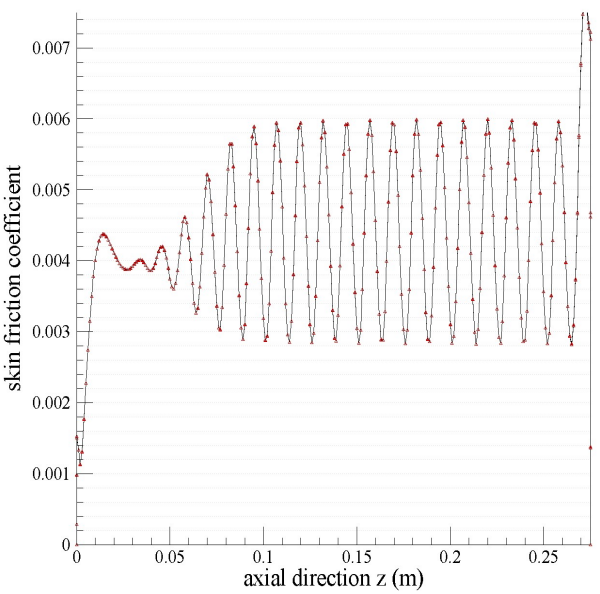

$\mathrm{Re}_{\mathrm{ax}}=3, \mathrm{Ta}=92.84$

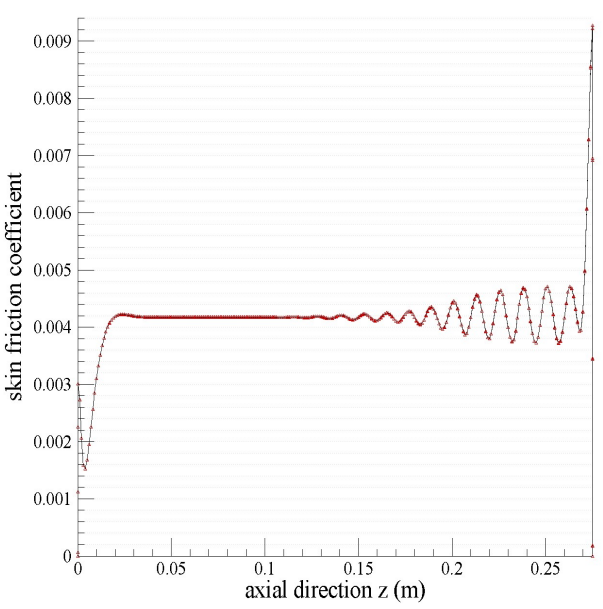

$\mathrm{Re}_{\mathrm{ax}}=6, \mathrm{Ta}=92.84$

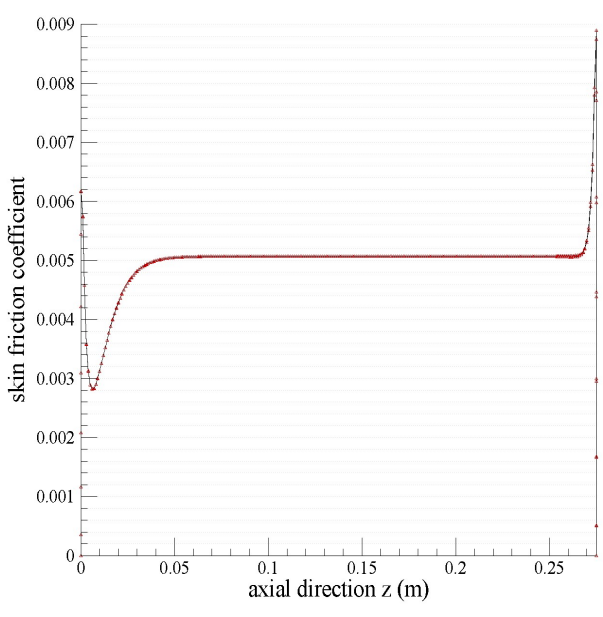

$\mathrm{Re}_{\mathrm{ax}}=12, \mathrm{Ta}=92.84$

Fig. 9. Evolution of friction coefficient versus the axial Reynolds Reax for $\mathrm{Ta}=92.84$.

\subsubsection{Effect of rotation on TVF mode reappearance}

At this stage of the study, evolution of the flow until reappearance of the Taylor vortices in the whole height of the column is targeted. 


\section{a) - Axial distribution of Taylor vortices}

\section{- Axial Reynolds number $\operatorname{Re}_{a x}=6$}

Figure 10 illustrates distribution of vortices versus the Taylor number with an imposed axial Reynolds number $\mathrm{Re}_{\mathrm{ax}}=6$. As shown in Fig. 10 (a), it seems that when rotation speed reaches a value corresponding to a Taylor number $\mathrm{Ta}=104.82$, the vortex reappears in the upper zone of the column. As the Taylor number increases, there is a progressive propagation of the piling vortices (Fig. 10 (b) and Fig. 10 (c)) until the complete occupation of the whole system lenght by the piled vortices for a value of the Taylor number $\mathrm{Ta}=124.28$ (Fig. 10 (d)).

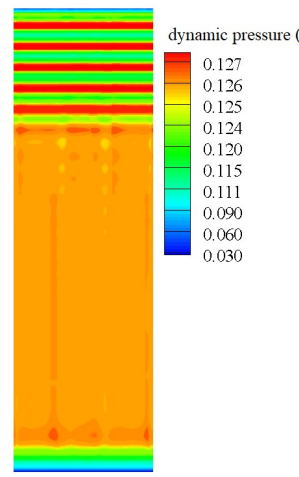

(a)

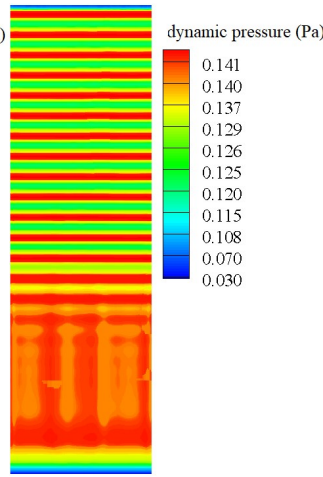

(b)
$\mathrm{Ta}=104.82$ $\mathrm{Ta}=110.81$

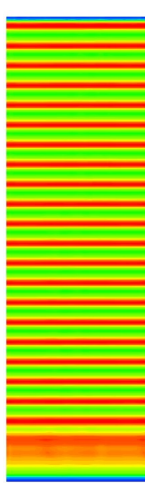

(c)

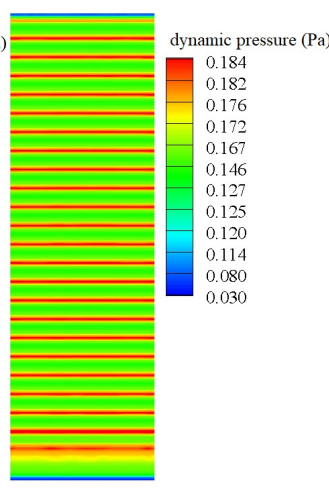

(d)
$\mathrm{Ta}=118.29$

$\mathrm{Ta}=124.28$ (TVF)

Fig. 10. Development of Taylor vortex versus the Taylor number for an axial Reynolds number $\mathrm{Re}_{\mathrm{ax}}=\mathbf{6}$.

\section{- Axial Reynolds number $\operatorname{Re}_{a x}=12$}

Figure 11 represents development of vortices versus Taylor number, for a fixed axial Reynolds number $\mathrm{Re}_{\mathrm{ax}}=12$. By increasing the control number up to $\mathrm{Ta}$ $=127.28$, the vortex piling in the column remains partial. We note that when $\mathrm{Ta}=142.25$ is attained, vortices piling region is confined in the interval $0.08 \leq z<0.1 \mathrm{~m}$. Beyond $\mathrm{z}=0.1 \mathrm{~m}$, the flow shifts to the WVF mode. These numerical results are consistent with those of Kristiawan et al. (2019) under the same conditions.

This reveals that the axial field tends to delay the onset of the TVF mode. The results obtained are comparable to those obtained by other authors, as reported in Table 2.

\begin{tabular}{|c|c|c|c|}
\hline Authors & $\eta$ & $\begin{array}{c}\text { Critical } \\
\text { Taylor } \\
\text { number } \\
T_{a c_{1}}^{*}\end{array}$ & $\begin{array}{c}\text { Critical } \\
\text { Taylor } \\
\text { number } T_{a c_{1}}\end{array}$ \\
\hline $\begin{array}{c}\text { Lueptow and } \\
\text { Wereley } \\
(1999)\end{array}$ & 0.83 & 60.7 & 123 \\
\hline present work & $\begin{array}{c}0.80 \\
4\end{array}$ & 61.32 & 124.28 \\
\hline
\end{tabular}

Table 2. Comparison of the critical Taylor numbers for an axial Reynolds number $R_{a x}=6$.

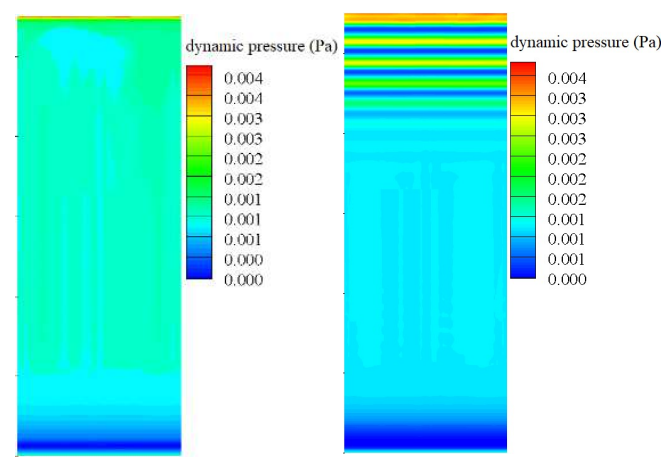

(a)

$\mathrm{Ta}=119.79$

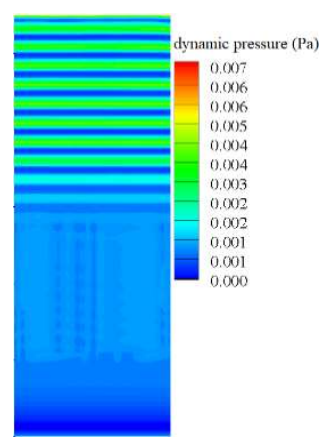

(c)

$\mathrm{Ta}=127.28$ (b)

$\mathrm{Ta}=121.29$

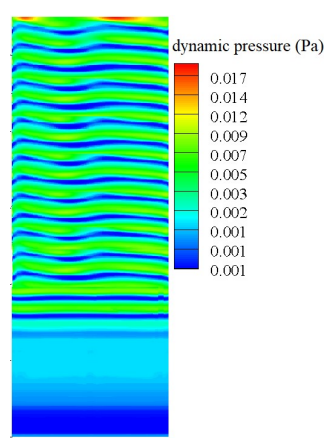

(d)
Fig. 11. Evolution of Taylor vortex for $\operatorname{Re}_{a x}=12$.

\section{b) - Distribution of vortices and azimuthal vorticity with and without an axial flow}

Figures 12 (a) and 12 (b) show distribution of Taylor vortices and streamlines at the bottom of the column in the absence and presence of axial flow at Reynolds number $\mathrm{Re}_{\mathrm{ax}}=6$. Comparing the two figures, it is found that under effect of intra-vortex flow, the vortices are oriented on the sidewalls and their sizes are reduced. The recorded wavelength $\lambda=9 \mathrm{~mm}$ is smaller compared to the standard case $\lambda=12.5 \mathrm{~mm}$. 
In addition, we note that there are no vortices at the column inlet in the region $\mathrm{z} \leq 0.02 \mathrm{~m}$.

In the absence of the axial field, the optimal values of the azimuthal vorticity are located in the center and the vicinity of the walls. Under influence of the axial field, maximum negative values are located on the side of the inner wall and maximum positive values are located in the center of the gap and on the side of the outer wall.

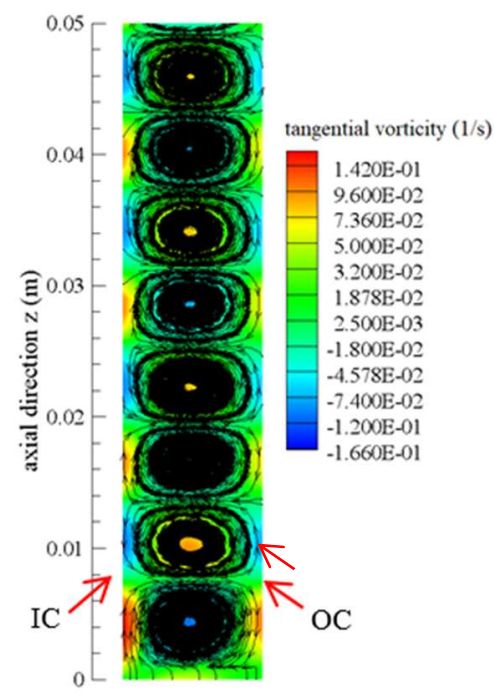

(a)

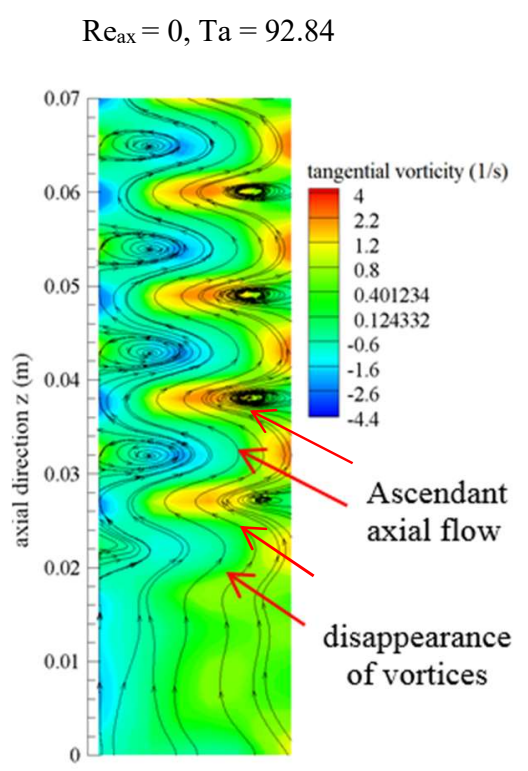

(b)

$\mathrm{Re}_{\mathrm{ax}}=6, \mathrm{Ta}=124.28$

Fig. 12. Details vortices distribution in the vicinity of the bottom region of the column.

\section{c) - Effect of axial flow on axial velocity distribution}

Figure 13 illustrates evolution of the axial velocity field along the azimuthal plane in the vicinity of the central part of the column, with and without axial flow. Figure 13(a) shows a shifted alternation of the maximum and minimum values of the axial velocity field relative to the central axis of the gap. We note in Fig. 13(b) that under influence of the axial field there is a predominance of positive values, consequently the distribution of the axial velocity field is asymmetric in the central axis of the gap.

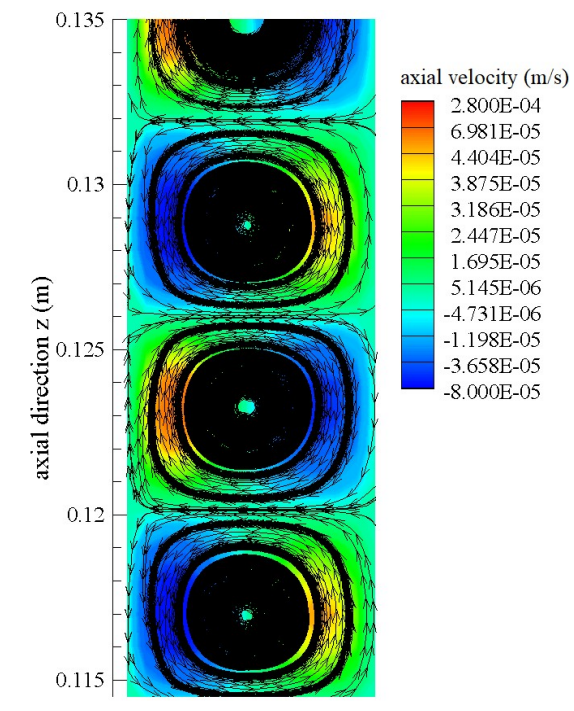

(a)

$$
\operatorname{Re}_{\mathrm{ax}}=0, \mathrm{Ta}=92.84
$$

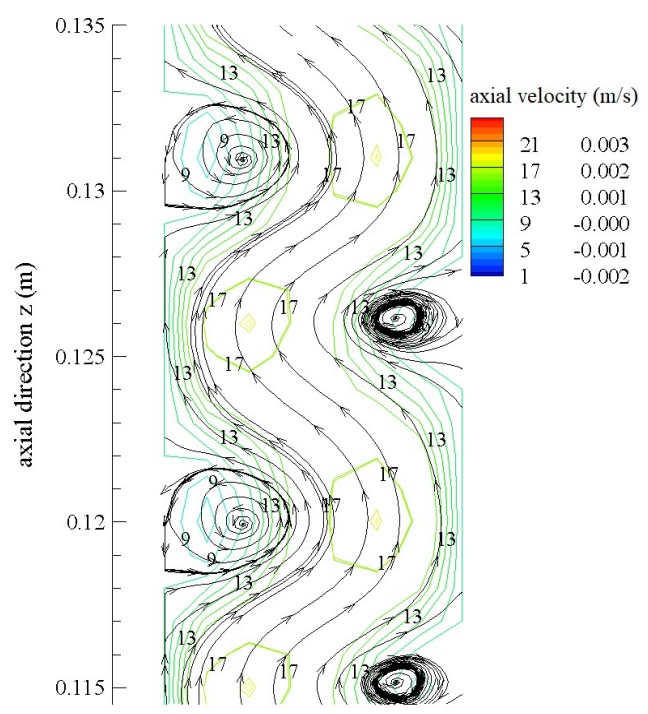

(b)

$$
\mathrm{Re}_{\mathrm{ax}}=6, \mathrm{Ta}=124.28
$$

\section{Fig. 13. Distribution of axial velocity in presence and absence of an axial flow.}

The profiles of the axial velocity field versus of the radial distance with and without an axial flow are shown in Fig. 14. The mean axial velocity is seen to be zero (stationary TVF mode), the axial velocity field profile shown in Fig. 14(a) is symmetric about the center of the gap. The mean axial velocity calculated at an axial distance $\mathrm{z}=130 \mathrm{~mm}$, is $v_{\mathrm{zm}}=$ 1.16 (propagative TVF mode in Fig. 14(b)). The 
ratio of derivation velocity to the mean base velocity is $\frac{V_{z m}}{V_{z}}=1.16$. This result conforms well to the values obtained by Recktenwald et al. (1993), Ng and Turner (1982), and DiPrima and Pridor (1979).

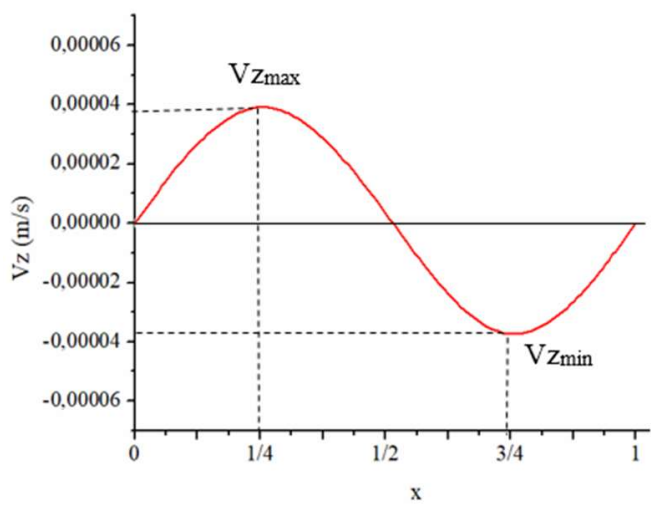

(a)

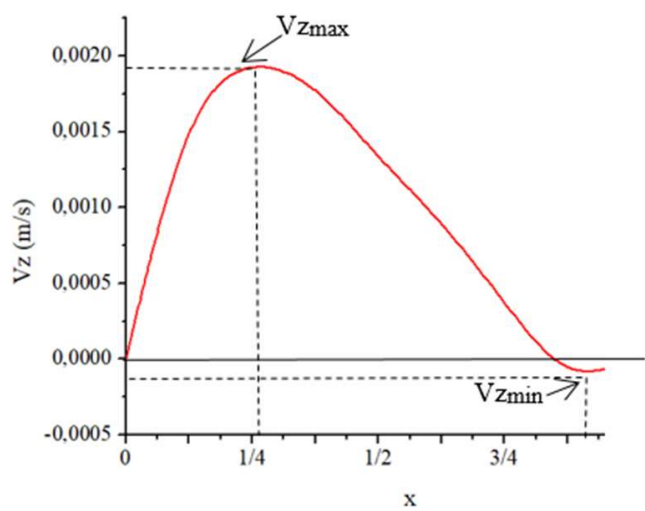

(b)

Fig. 14. Axial velocity profiles for TVF mode with and without an axial flow.

\section{Conclusion}

This work is devoted to the study of the stability of the Taylor-Couette flow system under the effect of an axial flow at non-vanishing Reynolds numbers. The study allowed us to obtain precise information on the evolution of the TVF mode in the absence and presence of an axial hydrodynamic field. First, for the standard case, (i.e., without axial field), we have tried to present details on the transition from laminar regime to TVF mode. We note that in the standard case for the same conditions, the values of the critical thresholds TVF and WVF correspond to those of other references. In the presence of an axial field, we have chosen two protocols to elucidate the influence of the axial field:

(a) The first protocol related to the relaminarization of the flow consists in varying the axial Reynolds number $\mathrm{Re}_{\mathrm{ax}}$ around the value of the critical Taylor number $\mathrm{T}_{\mathrm{ac}}$.

b) The second protocol concerns the opposite case, where the axial Reynolds number Reax is imposed and the Taylor number Ta is gradually increased until the TVF wave reappears.

Analysis of the results within the framework of the first protocol allowed access to information about the distribution of the streamlines as well as the azimuthal vorticity for different values of the Reynolds number. We note that for values of Reynolds number up to $\mathrm{Re}_{\mathrm{ax}}=6$, relaminarization of the flow remains partial. The regime becomes totally laminar when the value of Reynolds number $R_{a x}=$ 12 is attained. It appears that relaminarization of the flow depends essentially on the intensity of the axial flow, as it has been specified by references. It seems, that the intensity of the axial field influences the distribution of the friction coefficient at the external wall, where thefluctuating value is damped and stabilizes around $\mathrm{C}_{\mathrm{f}}=0.005$ corresponding to the value of Reynolds number $\operatorname{Re}_{\mathrm{ax}}=12$.

From the second protocol, we have highlighted the effect of the axial field on the onset of the first TVF mode. We note that for the value of the axial Reynolds number $\mathrm{Re}_{\mathrm{ax}}=12$, the distribution of the vortex piling at the-column level remains partial. In addition, we notice that for a large value of the Taylor number $\mathrm{Ta}=142.25$ the vortices are undulated (the WVF mode). It has been noticed, that for an axial Reynolds number $\mathrm{Re}_{\mathrm{ax}}=6$ and a Taylor number $\mathrm{Ta}=124.28$ the vortices settle completely on the whole column and that the intra-vortex flow tends to reduce the size of the vortices. Therefore the wavelength $\lambda=9 \mathrm{~mm}$ is smaller than the standard case $\lambda=12.5 \mathrm{~mm}$.

The vortex rollers translate with a deviation velocity $\mathrm{Vz}=1.16 \mathrm{~mm} / \mathrm{s}$. The ratio of the axial phase velocity to the mean base axial field velocity is well verified compared to the values obtained by Recktenwald et al. (1993), Ng and Turner (1982), and DiPrima and Pridor (1979)

\section{ACKNOWLEDGEMENTS}

The first author would like to thank Professor SOBOLIK for hosting him at the LaSIE laboratory of University of La Rochelle.

\section{REFERENCES}

Abdelali, A., H. Oualli, A. Rahmani, B. Merzkane and A. Bouabdallah (2019). Experiment and numerical simulation of Taylor - Couette flow controlled by oscillations of inner cylinder cross-section. Journal of the Brazilian Society of Mechanical Sciences and Engineering 41(6), 1-8.

Ali, M. E., D. Mitra, J. A. Schwille and R. M. Lueptow (2002). Hydrodynamic stability of a suspension in cylindrical Couette flow. Physics of Fluids 14 (3).

Andereck, C. D., S. S. Liu and H. L. Swinney (1986). Flow regimes in a circular Couette system with independently rotating cylinders. Journal of Fluid Mechanics 164, 155-183. 
Bouabdallah, A. (1980). Instabilités et turbulence dans l'écoulement de Taylor-Couette. Ph. D. thesis, INPL, Nancy, France.

Burkhalter, J. E. and E. L. Koschmieder (1973). Steady supercritical Taylor vortex flow. Journal of Fluid Mechanics 58 part 3, 547-560.

Chandrasekhar (1961). Hydrodynamic and hydromagnetic stability. Dover Publications.

Coles (1965). Transition in circular Couette flow. Journal of Fluid Mechanics 21,385-425.

DiPrima, R. C. and A. Pridor (1979). The stability of viscous flow between rotating concentric cylinders with an axial flow. Proceedings of the Royal Society of London. Series A, Mathematical and Physical Sciences 366, 555.

Drazin, F. and W. H. Reid (1981). Hydrodynamic stability. Cambridge University Press.

Fenstermacher, P. R., H. L. Swinney and J. P. Gollub (1979). Dynamical instabilities and transition to chaotic Taylor vortex flow. Journal of Fluid Mechanics 94, part1,103-128.

Hwang, J. Y. and K. S. Yang (2004). Numerical study of Taylor-Couette flow with an axial flow. Computers and Fluids 33, 97-118.

Kataoka, K., H. Doi and T. Komai (1977). Heat and mass transfer in Taylor vortex flow with constant axial flow rates. International Journal of Heat and Mass Transfer 20, 57-63.

Kristiawan, M., M. El Hassan, A. El Faye and V. Sobolík (2019). Experimental investigation of Taylor-Couette-Poiseuille flow at low Taylor and Reynolds numbers. PLoS ONE 14(4), 1-21.
Lueptow, R. M., A. Docter and K. Min (1992). Stability of axial flow in an annulus with a rotating inner cylinder. Physics of Fluids $A$ 4(11), 2446-2455.

Lueptow R. M. and S. T. Wereley (1999). Velocity field for Taylor-Couette flow with an axial flow. Physics of Fluids 11(12), 3637-3649.

Monfared, M. and E. Shirani (2016). Numerical and experimental study on the flow history effects of axial flow on the Couette-Taylor flow. Springer-Verlag Wien 227(7), 1999-2010.

$\mathrm{Ng}$, B. S. and E. R. Turner (1982). On the linear stability of spiral flow between rotating cylinders. Proceedings of the Royal Society of London. Series A, Mathematical and Physical Sciences 382, 83-102.

Ohmura, N., T. Suemasu and Y. Asamura (2005). Particle classification in Taylor vortex flow with an axial flow. Journal of physics: Conference Series (14), 64-71.

Recktenwald, A., M. Lucke, and H. W. Muller (1993). Taylor vortex formation in axial through-flow. Linear and weakly nonlinear analysis, Physical Review E 48(6), 4444.

Tsameret, A. and V. Steinberg (1994). Absolute and convective instabilities and noise-sustained structures in the Couette-Taylor system with an axial flow. Physical Review E, 49, 1291- 1308.

Wereley, S. T. and R. M. Lueptow (1998). Spatiotemporal character of non-wavy and wavy Taylor-Couette flow. Journal of Fluid Mechanics364, 59-80. 